Artículo científico

Volumen 31(2):291-309. Mayo-agosto, 2020

e-ISSN 2215-3608, doi:10.15517/am.v31i2.38387

http://www.revistas.ucr.ac.cr/index.php/agromeso

\title{
Flujos de metano en suelos con coberturas de pastos en el norte de Colombia ${ }^{1}$
}

\section{Methane fluxes in soils with pasture covers in the North of Colombia}

\author{
Manuel Espinosa-Carvajal², José Luis Contreras-Santos², Jorge Cadena-Torres², Judith del Carmen Martínez- \\ Atencia ${ }^{2}$, Camilo Ignacio Jaramillo-Barrios ${ }^{3}$, María del Pilar Hurtado ${ }^{4}$
}

1 Recepción: 1 de agosto, 2019. Aceptación: 11 de febrero, 2020. Este trabajo fue parte del proyecto "Producción intensiva de carne en pastos con diferente capacidad para inhibir la nitrificación y reducir las emisiones de gases de efecto invernadero", financiado por el Ministerio de Agricultura y Desarrollo Rural a través del Convenio No. 0542008, y se llevó a cabo en la Corporación Colombiana de Investigación Agropecuaria (AGROSAVIA), en el Centro Investigación Turipaná, Montería, Córdoba, Colombia.

2 Corporación Colombiana de Investigación Agropecuaria (AGROSAVIA), Centro de Investigación Turipaná, Km 13 Vía Montería-Cereté, Colombia. mespinosa@agrosavia.co (https://orcid.org/0000-0001-8939-5472); jlcontreras@agrosavia.co (autor para correspondencia, https://orcid.org/0000-0002-8179-3430); jcmartinez@agrosavia.co (https://orcid.org/0000-0002-8275-2956); jcadena@agrosavia.co (https:// orcid.org/0000-0002-5180-2893).

3 Corporación Colombiana de Investigación Agropecuaria (AGROSAVIA), Centro de Investigación Nataima, Km 9 vía Espinal-Chicoral, Tolima, Colombia. cijaramillo@agrosavia.co (https://orcid.org/0000-0002-8302-2736).

4 Universidad Nacional de Colombia, Sede Palmira. Carrera 13 \#12-00, Palmira, Colombia. pilyhurt@ gmail.com (https://orcid.org/0000-00016204-1797).

\section{Resumen}

Introducción. Los sistemas tradicionales de producción ganadera en Colombia se basan en el establecimiento de pasturas en grandes extensiones de tierra, que generan gases de efecto invernadero, como el metano. Objetivo. El objetivo del presente estudio fue vigilar los flujos mensuales de metano que se producen en las praderas con tres cubiertas de suelo y tres niveles de fertilización de nitrógeno. Materiales y métodos. Se realizó un monitoreo a los flujos de metano durante un año comprendido entre noviembre de 2014 y noviembre de 2015, en un suelo Vertic Endoaquepts, ubicado en el Valle medio del río Sinú, Colombia. Se usó un diseño de bloques completos al azar, en arreglo en parcelas divididas, con dos repeticiones. Las parcelas principales constituidas por dos gramíneas (Brachiaria humidicola CIAT679 y Panicum maximum cv. Tanzania) y una con suelo descubierto, y las subparcelas por tres niveles de fertilización nitrogenada $\left(0,150,300 \mathrm{~kg} \mathrm{~N} \mathrm{ha}^{-1}\right)$. Adicionalmente, se evaluaron las propiedades fisicoquímicas del suelo. Resultados. Durante la mayor parte del año (época de lluvias y seca) ocurrió oxidación de metano, asociado con la textura franca y porosidad del suelo en el área de estudio, que permitió la difusión libre de gases en el suelo. Los análisis de correlación mostraron estrecha relación entre los flujos de metano, porosidad, humedad, densidad aparente del suelo y la temperatura ambiente, sugiriendo a estos parámetros como los principales factores que afectan el flujo mensual de metano. Conclusiones. Los flujos de metano fueron independientes del tipo material vegetal y de la fertilización nitrogenada evaluada. Estos flujos dependieron de la época del año (seca y lluviosa) y exhibieron un balance anual negativo en el área de estudio, lo que sugiere que estos sistemas tienen las condiciones para comportarse como sumideros de metano durante la mayor parte del año.

Palabras clave: gases de efecto invernadero, propiedades del suelo, Panicum, Brachiaria, producción ganadera. 


\begin{abstract}
Introduction. Traditional livestock production systems in Colombia are based on the establishment of pastures on large tracts of land, which generate greenhouse gases such as methane. Objective. The objective of the present study was to monitor the monthly methane fluxes that occur in meadows with three soil covers and three levels of nitrogen fertilization. Materials and methods. Methane fluxes were monitored for a year from November 2014 to November 2015, on a Vertic Endoaquepts soil, placed in the middle valley of the Sinu river, Colombia. A full block design was used at random, arranged in divided plots, with two replications. The main plots constituted of two grasses (Brachiaria humidicola CIAT679 and Panicum maximum cv. Tanzania) and one with bare soil, and the subplots by three nitrogen fertilization levels $\left(0,150,300 \mathrm{~kg} \mathrm{~N} \mathrm{ha}^{-1}\right)$. Additionally, soil physicochemical properties were evaluated. Results. During most of the year (rainy and dry season) methane oxidation occurred, associated with the loam texture and porosity of the soil in the study area, which allowed the free diffusion of gases in the soil. Correlation analyzes showed a close relationship between methane fluxes, porosity, moisture, soil bulk density, and ambient temperature, suggesting these parameters as the main factors that affect the monthly methane flow. Conclusions. Methane fluxes were independent of the type of plant material and nitrogen fertilization evaluated. These flows depended on the time of year (dry and rainy) and exhibited a negative annual balance in the study area, which suggests that these systems have the conditions to behave as methane sinks during most of the year.
\end{abstract}

Keywords: greenhouse gases, soil properties, Panicum, Brachiaria, livestock production.

\title{
Introducción
}

Las actividades antropogénicas son las principales causantes de las emisiones de los gases de efecto invernadero hacia la atmósfera en los últimos dos siglos y medio (1750 - 2011), a causa del crecimiento demográfico y económico (Pachauri et al., 2014). En 2010, se reportaron las mayores emisiones de gases de efecto invernadero (GEI) por causas antropogénicas con 49 Gt GEI, con un incremento anual del 2,2 \% (Pachauri et al., 2014). El metano es el GEI con mayor aumento, con alrededor del $150 \%$, mostrando las mayores emisiones a partir del año 2007, debido al aumento de la temperatura causante del deshielo de los polos y a la actividad pecuaria (Pachauri et al., 2014; Hernández-Medrano y Corona, 2018). Los sistemas ganaderos a nivel global representan el $14 \%$ de las emisiones de GEI, y la ganadería bovina es responsable de 2/3 de ese total, gran parte debido a emisiones de $\mathrm{CH}_{4}$, causadas por la fermentación ruminal, desechos de animales y cambio en el uso del suelo (Lassey, 2008; Charmley et al., 2016; US EPA, 2017; FAO, 2018).

En Colombia como en otras partes del mundo, la expansión de ganadería se ha basado en la continua conversión de áreas que antiguamente estaban cubiertas por bosques, rastrojos, ciénagas y pantanos, en praderas aptas para la producción de leche y carne. En la actualidad, las estadísticas indican que la producción ganadera en Colombia ocupa aproximadamente 34 millones de hectáreas, lo que representa el $30 \%$ del área total adecuada para la producción agrícola en el país. El modelo de producción practicado por la mayor parte de los ganaderos es preferencialmente extensivo con baja carga animal y baja eficiencia en el uso de la tierra (Murgueitio y Muhammad, 2009; Murgueitio, 2011; Mora et al., 2017).

En el Valle medio del río Sinú, en el norte de Colombia, existe un sistema de producción de carne, en donde las áreas que antiguamente estaban cubiertas por cuerpos de agua, ciénagas y pantanos, fueron convertidas a la producción ganadera, mediante sistemas de drenajes. Los suelos en esta región son de origen aluvial y alta fertilidad, lo que ha conllevado al establecimiento de sistemas de producción de carne que han pasado a ser cada vez más intensivos, basados en el alimento que proporcionan pasturas mejoradas, complementadas con fertilizantes 
nitrogenados, con lo cual han logrado aumentar la carga animal y obtener una mayor producción de carne por hectárea, con un aumento de las emisiones de gases de efecto invernadero (GEI). Las investigaciones realizadas por diferentes autores (Lee et al., 2009; Aronson y Helliker, 2010; Hristov et al., 2013; Clark, 2016; Fang et al., 2014; Yang et al., 2017; Li et al., 2018), han reportado que el incremento de aplicaciones y/o dosis de fertilizantes nitrogenados podría afectar los procesos de oxidación del metano en el suelo, cantidades superiores a $100 \mathrm{~kg} \mathrm{~N} \mathrm{ha}^{-1}$ año ${ }^{-1}$, inhiben la actividad de los metanotrófos en el suelo. Existe interés de conocer el comportamiento de los flujos de metano en los suelos de Valle medio del río Sinú, debido a que, por su origen y características, se podrían estar generando emisiones importantes de GEI hacia la atmósfera. Es bien conocido que la actividad de los organismos metanógenicos es fuertemente afectada por las condiciones de humedad del suelo y la variación en los niveles de oxígeno (Hao et al., 1988; Scharffe et al., 1990; Sanhueza et al., 1994; Sanhueza y Donoso, 2006; Sanhueza, 2007).

Se ha encontrado que los contenidos de humedad adecuados para la absorción de metano en el suelo corresponden a la capacidad de campo, una condición bajo la cual los procesos de oxidación no se ven afectados (Boeckx et al., 1997; Hou et al., 2000; Pereyra, 2009). Altas precipitaciones reducen la aireación en los suelos y aumentan los flujos de metano a la atmósfera (Castro et al., 2000; Kähkönen et al., 2002). Se ha reconocido que el aspecto más crítico a destacar en los estudios de flujos de metano está relacionado con los contenidos de humedad en el suelo, debido a sus efectos sobre la oxidación del metano (Visscher et al., 2007). Durante las temporadas de lluvias, especialmente en las regiones donde ocurren altas precipitaciones, el metano se filtra a la atmósfera, debido a las condiciones anaeróbicas que se desarrollan en el suelo. Estos flujos de metano solo disminuyen y es negativo (captura) cuando se reduce la saturación, a causa de la disminución de la precipitación (mayor presencia de oxígeno), favoreciendo los procesos oxidativos para la absorción o secuestro de metano por las bacterias aeróbicas (Klemedtsson y Klemedtsson, 1997; Kammann et al., 2001; Dunfield, 2007; Reay et al., 2007; Visscher et al., 2007; Ferreira, 2008).

El presente estudio se realizó con el objetivo de estudiar la dinámica de los flujos de metano en suelos ganaderos cubiertos con praderas, pasturas mejoradas y con fertilización nitrogenada. Se asumió que, por haber estado cubierto por cuerpos de agua en el pasado, por su origen fluvio lacustre, con drenajes que podrían ser imperfectos, los suelos del Valle medio del río Sinú podrían estar favoreciendo la actividad de microorganismos metanogénicos, generando cantidades importantes de metano hacia la atmósfera.

\section{Materiales y métodos}

\section{Locación}

La presente investigación se llevó a cabo entre el 15 de noviembre de 2014 y el 15 de noviembre de 2015 en el Centro de Investigación Turipaná, representativo de los suelos y el paisaje del Valle medio del río Sinú, en el Norte de Colombia. Este centro de investigación pertenece a la Corporación Colombiana de Investigación Agropecuaria (AGROSAVIA) y se encuentra localizado en el municipio de Cereté, departamento de Córdoba, con coordenadas geográficas $8^{\circ} 51^{\prime} 3,9^{\prime \prime} \mathrm{N}$ y $75^{\circ} 48^{\prime} 3,3$ ” W, a $14 \mathrm{msnm}$. Presenta una temperatura media anual de $28^{\circ} \mathrm{C}$, humedad relativa $87 \%$ y precipitación media anual de $1200 \mathrm{~mm}$, distribuida en una época lluviosa que ocurre entre abril y noviembre, seguida por una época seca entre los meses de diciembre a marzo. De acuerdo con Holdridge (2000), el área de estudio hace parte de la zona agroecológica del bosque seco tropical (BsT).

El área de estudio posee suelos de planicie, correspondiente a áreas bajas dentro de la posición, su material de origen es fino o pesado y se forman en bacines. El drenaje natural es imperfectamente drenado en las áreas más altas, pobremente y muy pobremente drenado en las más bajas. Los grupos texturales predominantes son francoarcilloso, franco-limoso y franco-arcillo-limoso, con mayor presencia del primer grupo. De acuerdo con USDA (2014) el suelo bajo estudio correspondió a un Vertic Endoaquepts régimen de humedad acuíco y temperatura 
isohipertérmico con secuencia de horizontes $\mathrm{Ap}, \mathrm{Bx}, \mathrm{Bg}$ y Cg. Las características del suelo del área de estudio, se muestran en el Cuadro 1.

Cuadro 1. Descripción de perfil de suelo en el sitio donde se evaluó el flujo de metano en suelo con cobertura. Centro de Investigación Turipaná, Corporación Colombiana de Investigación Agropecuaria (AGROSAVIA). 2014-2015.

Table 1. Description of soil profile at the site where the methane fluxes in covered soil were evaluated. Turipaná Research Center, Corporación Colombiana de Investigación Agropecuaria (AGROSAVIA). 2014-2015.

\begin{tabular}{|c|c|}
\hline Horizonte & Descripción \\
\hline$A p(0-10 \mathrm{~cm})$ & $\begin{array}{l}\text { Color en húmedo gris oscuro ( } 5 \text { Y 4/1), con } 25 \% \text { moteados de color pardo amarillento (10 YR } \\
5 / 8 \text { ); textura fina (franco arcillosa); estructura bloques subangulares medios con grado moderado; } \\
\text { consistencia en seco dura, en húmedo firme y en mojado pegajoso y plástico; distribución de poros } \\
\text { en cantidades pocas de tamaños finos a medios; con alta presencia de raíces de tamaños medios a } \\
\text { finos localizadas a lo largo del perfil, ubicadas en grietas y ancho del horizonte, frecuente actividad } \\
\text { de macro invertebrados (lombrices, hormigas, etc), límite de horizonte claro. }\end{array}$ \\
\hline$B x(10-25 \mathrm{~cm})$ & $\begin{array}{l}\text { Color en húmedo gris pardusco claro ( } 2,5 \text { Y 6/2), con moteados de color pardo amarillentos ( } 10 \text { YR } \\
5 / 6) \text {; textura franco arcillosa, estructura en bloque subangulares de clases finas a medias, de grado } \\
\text { moderado a débil; consistencia en seco muy dura, húmedo firme, en mojado pegajosa y plástica; } \\
\text { abundantes poros de tamaños finos a medios, con poca presencia de macroorganismos, con presencia } \\
\text { de raíces de tamaños finas a muy finas, con características fragipán. }\end{array}$ \\
\hline Bg $(25-47 \mathrm{~cm})$ & $\begin{array}{l}\text { Color en húmedo grisáceo oliva claro ( } 5 \mathrm{Y} 6 / 2) \text {, con } 30 \% \text { de moteados de color rojo amarillento } \\
(5 \mathrm{YR} 4 / 6) \text {; textura arcillosa, estructura en bloques subangulares de clase fina con grado débil; } \\
\text { consistencia en seco muy dura, húmedo muy firme, en mojado muy pegajosa y muy plástica; poca } \\
\text { presencia de poros, principalmente se evidencia poros de tamaño finos a muy finos, poca presencia } \\
\text { de raíces, escasa o poco presencia de macroorganismos, límite de horizonte difuso, presencia de } \\
\text { procesos de oxido reducción. }\end{array}$ \\
\hline $\mathrm{Cg}(47-100 / \mathrm{x} \mathrm{cm})$ & $\begin{array}{l}\text { Color en húmedo pardo rojizo ( } 5 \text { YR } 4 / 4) \text {, alta presencia de moteados ( }>50 \% \text { ) de color rojo }(2,5 \\
\text { YR 4/6); textura franco limos (FL); sin estructura (suelta), consistencia en seco ligeramente dura, en } \\
\text { húmedo friable y en mojado ligeramente pegajosa y ligeramente plástica; raíces finas escasas, límite } \\
\text { difuso; pH 5,2, reacción ácida. }\end{array}$ \\
\hline
\end{tabular}

\section{Tratamientos}

El experimento se estableció en potreros de producción de ganadería de carne, en un área de 12,0 ha, los cuales presentaban cobertura con las gramíneas Brachiaria humidicola cv. CIAT679 y Panicum maximum cv. Tanzania, las cuales habían sido establecidas al azar para procesos de investigación previos. Los potreros o parcelas principales (PP) se dividieron en subparcelas (SP), en las cuales se aplicaron al azar tres niveles de fertilización nitrogenada $\left(0,150\right.$ y $\left.300 \mathrm{~kg} \mathrm{ha}^{-1}\right)$. La fuente de nitrógeno utilizada fue urea $\left(\mathrm{CO}\left(\mathrm{NH}_{2}\right)_{2}\right)$, con una concentración de $46 \%$ de $\mathrm{N}$. Al lado de las pasturas o parcelas principales, se dejaron parcelas a las cuales se les removió completamente la pastura establecida, para dejar el suelo al descubierto, en las cuales se aplicaron también al azar los tres niveles de fertilización nitrogenada. Lo anterior permitió la evaluación de nueve tratamientos, consistentes en los tres tipos de cobertura y los tres niveles de fertilización nitrogenada (Cuadro 2).

Las parcelas principales, correspondiente a las pasturas, tenían un área de 3,0 ha y las subparcelas un área de 1,0 ha. Las parcelas principales (PP) con suelo descubierto tenían un área de $3000 \mathrm{~m}^{2}$ y las $\mathrm{SP} 1000 \mathrm{~m}^{2}$. Las parcelas principales con cobertura de gramíneas fueron divididas en doce potreros de $2500 \mathrm{~m}^{2}$ cada uno, para pastoreo rotacional, en los cuales se ingresaron doce terneros de la raza Brahman, con un peso de entrada entre 200 y 250 $\mathrm{kg}_{\text {animal }}{ }^{-1}$. Lo anterior arrojó una carga animal de 4 animales ha ${ }^{-1}$. 
Cuadro 2. Tratamientos establecidos para el monitorear los flujos de metano del suelo con diferentes coberturas de pastura (Brachiaria humidicola cv. CIAT679, Panicum maximum cv. Tanzania) en el valle medio del río Sinú, Colombia, y el efecto de la fertilización nitrogenada. $2014-2015$.

Table 2. Treatments established to monitor soil methane fluxes with different pasture covers (Brachiaria humidicola cv. CIAT679, Panicum maximum cv. Tanzania) at the middle valley of the Sinú river, in Colombia, and the effect of nitrogen fertilization. 2014-2015.

\begin{tabular}{ccc}
\hline Tratamientos No. & Cobertura de suelo & $\begin{array}{c}\text { Niveles de fertilización nitrogenada } \\
\left(\mathbf{k g ~ h a}^{-1} \mathbf{a n ̃ o}^{-1}\right)\end{array}$ \\
\hline 1 & Brachiaria humidícola cv. CIAT679 $(\mathrm{BH})$ & 0 \\
2 & Brachiaria humidícola cv. CIAT679 $(\mathrm{BH})$ & 150 \\
3 & Brachiaria humidícola cv. CIAT679 $(\mathrm{BH})$ & 300 \\
4 & Panicum maximum cv. Tanzania (PM) & 0 \\
5 & Panicum maximum cv. Tanzania (PM) & 150 \\
6 & Panicum maximum cv. Tanzania (PM) & 300 \\
7 & Suelo descubierto & 0 \\
8 & Suelo descubierto & 150 \\
9 & Suelo descubierto & 300 \\
\hline
\end{tabular}

\section{Diseño experimental}

Se utilizó un diseño experimental en bloques completos al azar con arreglo en parcelas divididas, donde la parcela principal estaba constituida por las coberturas de pasturas (Brachiaria humidicola CIAT 679, Panicum maximum cv Tanzania y suelo descubierto), y la subparcela por tres niveles de fertilización nitrogenada $(0,150$ y $\left.300 \mathrm{~kg} \mathrm{~N} \mathrm{ha}^{-1} \mathrm{año}^{-1}\right)$, con dos repeticiones. El factor de bloqueo correspondió al drenaje del suelo.

\section{Variables de respuesta}

\section{Ambientales}

Se registraron variables ambientales como la precipitación $\left(\mathrm{mm} \mathrm{mes}^{-1}\right)$, humedad relativa (\%), evaporación $\left(\mathrm{mm} \mathrm{día} \mathrm{a}^{-1}\right)$, temperatura $\left({ }^{\circ} \mathrm{C}\right)$ máxima, mínima y media registradas diariamente a las 07,13 y $18 \mathrm{~h}$, usando una estación climatológica localizada en el área de influencia del experimento.

\section{Propiedades del suelo}

Se determinaron las propiedades físicas y químicas del suelo, mediante muestras tomadas a 0,20 m de profundidad, al inicio y final del experimento en cada una de las unidades experimentales. Las muestras de suelo se procesaron en el Laboratorio de Suelo de la Universidad de Córdoba, donde se realizaron determinaciones químicas del suelo. El pH se obtuvo por el método potenciométrico con una relación 1:1 (p/v). La materia orgánica se determinó mediante el método de oxidación Walkley-Black y el fósforo (P) mediante el método de Olsen modificado. El azufre (S) se extrajo con fosfato de calcio monobásico y se estimó por colorimetría (IGAC, 2006).

Las propiedades físicas del suelo se determinaron en el Laboratorio de Suelos del Centro Internacional de Agricultura Tropical (CIAT) La densidad real se obtuvo por el método del picnómetro (Burt, 2014), la densidad aparente (Da) por el método del cilindro sin cambios (Burt, 2014), la porosidad total (Pt) por el método de Bernabé 
y Maineult (2015), y la conductividad hidráulica saturada (CH) por el método propuesto por el Instituto Geográfico Agustín Codazzi (IGAC, 2006), porcentaje de la porosidad ocupada por agua (water filled porosity space, WFPS) (ecuación 1).

$$
\text { WFPS }=(H u m * D a) / P t \quad \text { Ecuación } 1
$$

Donde:

Hum: humedad gravimétrica $\left(\mathrm{g} \mathrm{g}^{-1}\right)$.

Da: densidad aparente $\left(\mathrm{g} \mathrm{cm}^{-3}\right)$.

Pt: porosidad total $\left(\mathrm{g} \mathrm{g}^{-1}\right)$.

Todos los análisis físicos y químicos del suelo se realizaron con base en los protocolos establecidos por IGAC (2006).

\section{Monitoreo del nivel freático}

Se realizó un monitoreo del nivel freático del suelo dentro del área de evaluación, mediante una red constituida por veinticuatro de pozos de observación freatimétrica, establecidos en cuadrículas cada $10 \mathrm{~m}$, a una profundidad 2,3 $\mathrm{m}$. Para los pozos se utilizaron tubos perforados de PVC de 2 pulgadas de diámetro, con perforaciones de $4 \mathrm{~mm}$ en espiral desde la base hasta $0,20 \mathrm{~cm}$ de la parte superior. Las observaciones de la profundidad del nivel freático se realizaron con periodicidad semanal, durante diez meses (febrero a noviembre 2015).

\section{Flujo de metano $\left(\mathrm{CH}_{4}\right)$}

Se realizó un monitoreo mensual de los flujos de metano entre noviembre de 2014 y noviembre de 2015, lo cual incluyó tanto la época de lluvias como la época seca. Para el monitoreo se utilizó la metodología de las cámaras cerradas estáticas, descrita por Rondón (2000); Montenegro y Abarca (2000), la cual había sido previamente estandarizada y validada por Chu et al. (2007). Para ello, se establecieron cámaras cerradas estáticas, por duplicado en cada unidad experimental, las cuales constaban de dos cilindros de PVC, de $25 \mathrm{~cm}$ de diámetro, unidos entre sí por medio de una banda de caucho (Figura 1). El primer cilindro, de 7,5 cm de altura, se insertó en el suelo al inicio del experimento, a una profundidad de $5,5 \mathrm{~cm}$. El segundo cilindro, de $10 \mathrm{~cm}$ de altura, se colocó al momento de la medición, en la parte superior del primero, ajustándolo con la banda de caucho. Este segundo cilindro poseía una tapa en la parte superior para asegurar el cierre hermético de la cámara, pero disponía de dos agujeros o septum, cubiertos con tapones de goma, uno de los cuales se usaba para tomar las muestras de gases y el otro, para registrar la temperatura del aire dentro de la cámara. Las mediciones se realizaron mensualmente, a las 10:00 am; con jeringas de $20 \mathrm{ml}$ se obtuvo una muestra de los gases dentro de la cámara. En cada cámara se tomaron muestras a los 0 (con la cámara descubierta), 15 y 45 minutos después de colocado el segundo cilindro. De la muestra de gases se descartaron $5 \mathrm{ml}$ y los restantes $15 \mathrm{ml}$ se depositaron en viales de $15 \mathrm{ml}$ de capacidad, herméticamente cerrados, previamente liofilizados y empacados al vacío (Rondón, 2000).

Cada vial con la muestra de gases se etiquetó, dentro de las $24 \mathrm{~h}$ siguientes a la toma de las muestras, se envió al Laboratorio de Servicios Ambientales del Centro Internacional de Agricultura Tropical (CIAT), donde se determinó la concentración de metano en un cromatógrafo de gases, equipado con un detector de ionización de llama (FID) (Capa, 2015). La lectura obtenida, junto con la temperatura del aire tomada en el momento del muestreo, las dimensiones de la cámara de muestreo y la ley de gases ideales, se utilizaron para calcular la densidad molar de metano en la muestra. 


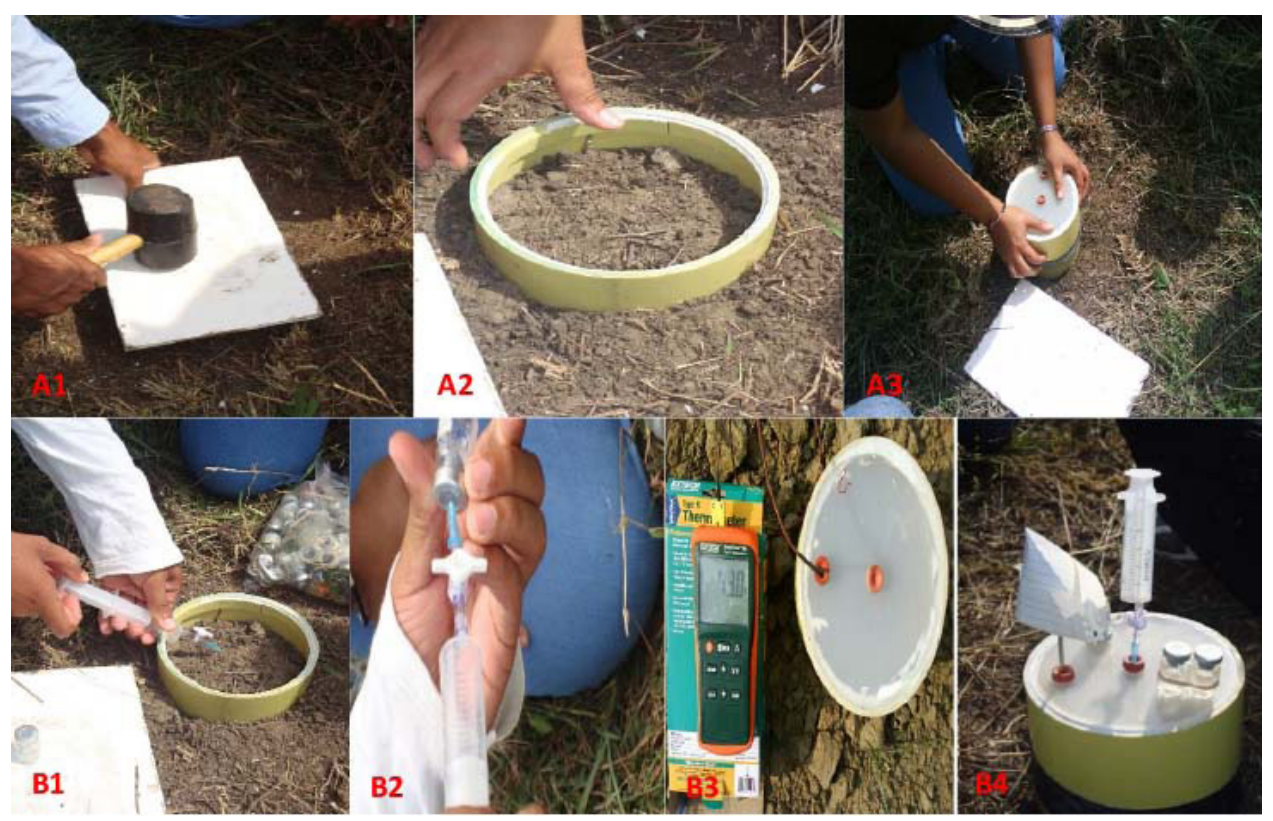

Figura 1. Cámaras utilizadas para monitorear los flujos de metano del suelo con diferentes coberturas de pastos: a) instalación de cilindros y cámaras de muestreo; b) proceso de muestreo. A1: instalación de cilindro; A2: cilindro instalado; A3: cilindro con cámara de muestreo; B1: toma de muestra inicial de gases; B2: traspaso de aire de la jeringa al vacutainer; B3: determinación de la temperatura del suelo; B4: toma de muestra de aire de la cámara cerrada. Valle medio del río Sinú, Córdoba, Colombia. 2014-2015.

Figure 1. Chambers used to monitor soil methane fluxes with different pasture covers: a) installation of cylinders and sampling chambers; b) sampling process. A1: cylinder installation; A2: installed cylinder; A3: cylinders with sampling chamber; B1: taking of initial sample of gases; B2: transfer of air from syringe to vacutainer; B3: determination of soil temperature; B4: air sample taken from the closed chamber. Middle valley of the Sinú river, Cordoba, Colombia. 2014-2015.

Los flujos de metano se calcularon utilizando las concentraciones obtenidas en los tiempos 0, 15, 30 y 45 min. Para este propósito, primero se revisó la relación de linealidad en las cuatro lecturas de cada muestra y se obtuvo la pendiente de regresión. Una vez verificada la linealidad de las observaciones, se utilizó la siguiente relación para calcular los flujos de metano (ecuaciones 2 y 3 ):

$$
F=\frac{d x}{d t} \times \frac{V M}{A V m} \quad \text { Ecuación } 2
$$

Donde:

$\mathrm{F}=$ flujo de metano $\left(\mathrm{mg} \mathrm{m}^{-2} \mathrm{~h}^{-1}\right)$.

$\mathrm{dx} / \mathrm{dt}=$ pendiente de la relación lineal entre la concentración de $\mathrm{CH}_{4}$ y el tiempo transcurrido $\left(\mathrm{ppm} \mathrm{min}{ }^{-1}\right.$ ).

$\mathrm{V}=$ volumen ocupado por el gas (1).

$\mathrm{A}=$ área de la cámara $\left(\mathrm{m}^{2}\right)$.

$\mathrm{M}=$ masa de $\mathrm{C}$ por cada mol de $\mathrm{CH}_{4}$.

$\mathrm{Vm}=$ volumen molecular del gas. 
$V m=n R T / P \quad$ Ecuación 3

Donde:

$\mathrm{n}=$ mol del gas.

$\mathrm{R}=$ constante de ley de gases ideales $\left(0,0820574587 \mathrm{~L} \mathrm{~atm} \mathrm{~K}^{-1} \mathrm{~mol}^{-1}\right)$.

$\mathrm{T}=$ temperatura dentro de la cámara $(\mathrm{K})$.

$\mathrm{P}=$ presión en atmósfera (atm).

Los resultados se presentaron como valores positivos o negativos, que representaron flujos de metano hacia la atmósfera en el primer caso, o hacia el suelo en el segundo caso (oxidación o absorción de metano).

\section{Análisis estadístico}

Se realizaron análisis de varianza (ANOVA) a través de modelos lineales generales y mixtos. La prueba LSD de Fischer se utilizó para comparar las medias entre tratamientos, con un $\alpha=0,05$. Para evaluar el cumplimiento de los supuestos de normalidad y homogeneidad se utilizaron gráficos de qq plot, histogramas y gráficos de cajas de residuos. La homogeneidad se evaluó utilizando gráficos de residuos versus datos esperados. Se seleccionó el modelo con el menor AIC, BIC y la máxima log verosimilitud (LogLik). Las variables ambientales y edáficas se sometieron a análisis de correlación de Pearson y componentes principales, con el fin de determinar la asociación de variables relacionadas con los flujos de metano. Todos los análisis estadísticos se realizaron en el software estadístico Infostat 2018 (Di-Rienzo et al., 2018) y R versión 3.4.4 usando los paquete nlme y FactoMineR (Lê et al., 2008; Pinheiro et al., 2018; R Core Team, 2018).

\section{Resultados}

\section{Condiciones ambientales}

Las condiciones ambientales durante el período experimental se muestran en la Cuadro 3. De acuerdo con el comportamiento particular del clima en el año de estudio, se podría considerar que la mayor parte de las mediciones del flujo de metano se realizaron cuando prevalecían condiciones secas, con precipitaciones inferiores a 100 por mes. Al respecto, Guzmán et al. (2014), han establecido que, para la costa norte de Colombia, la época seca corresponde a períodos mensuales que reciben precipitaciones totales mensuales inferiores a los $100 \mathrm{~mm}$. Bajo esta premisa, las muestras de flujo de metano obtenidos en los meses de noviembre y diciembre de 2014, y enero, febrero, marzo, abril, mayo y noviembre de 2015 , podrían considerarse que corresponden a periodos prevalentemente secos. De igual forma, las muestras tomadas en los meses de junio, julio, agosto, septiembre y octubre de 2015, ocurrieron durante lo que podría considerarse época húmeda. Las mayores precipitaciones se presentaron en los meses de mayo y julio de 2015.

\section{Nivel freático}

La profundidad de la capa freática se observó siempre a más de $50 \mathrm{~cm}$ de profundidad, sin ascenso del agua subsuperficial, incluso durante la temporada de lluvias (Figura 2). Esto indica que durante todo el período experimental prevalecieron condiciones drenadas en el suelo, sin ascensos del nivel freático, lo cual favoreció la 
Cuadro 3. Condiciones ambientales durante el período experimental para monitorear los flujos de metano del suelo con diferentes coberturas de pastos (Brachiaria humidicola cv CIAT679, Panicum maximum cv Tanzania), en el valle medio del río Sinú en Colombia. $2014-2015$.

Table 3. Environmental conditions during the experimental period to monitor soil methane fluxes with different pasture covers (Brachiaria humidicola cv CIAT679, Panicum maximum cv Tanzania), in the middle valley of the Sinú river in Colombia. 2014 - 2015.

\begin{tabular}{cccccccc}
\hline Muestra No & Días & Mes & $\begin{array}{c}\text { Precipitación total } \\
\left(\mathbf{m m ~ m e s}^{-1}\right)\end{array}$ & $\begin{array}{c}\text { Temperatura } \\
\text { del aire }\left({ }^{\circ} \mathbf{C}\right)\end{array}$ & $\begin{array}{c}\text { Humedad } \\
\text { Relativa }(\boldsymbol{\%})\end{array}$ & $\begin{array}{c}\text { Evaporación } \\
\left(\mathbf{m m} \text { día }^{-1}\right)\end{array}$ & Época \\
\hline 1 & 0 & Noviembre & 54,4 & 27,92 & 69,50 & 3,26 & Seca \\
2 & 30 & Diciembre & 43,9 & 28,51 & 65,86 & 4,24 & Seca \\
3 & 60 & Enero & 0,0 & 28,16 & 63,65 & 4,79 & Seca \\
4 & 90 & Febrero & 5,0 & 28,80 & 66,93 & 5,09 & Seca \\
5 & 120 & Marzo & 74,0 & 29,19 & 68,47 & 4,85 & Seca \\
6 & 150 & Abril & 87,4 & 29,19 & 71,98 & 4,49 & Seca \\
7 & 180 & Mayo & 245,4 & 28,76 & 72,48 & 4,24 & Seca \\
8 & 210 & Junio & 107,2 & 27,80 & 73,75 & 3,16 & Lluviosa \\
9 & 240 & Julio & 286,0 & 27,90 & 74,31 & 3,43 & Lluviosa \\
10 & 270 & Agosto & 165,4 & 27,57 & 74,75 & 3,39 & Lluviosa \\
11 & 300 & Septiembre & 193,8 & 27,28 & 74,15 & 3,64 & Lluviosa \\
12 & 330 & Octubre & 156,3 & 27,72 & 73,37 & 3,65 & Lluviosa \\
13 & 360 & Noviembre & 71,8 & 27,02 & 74,11 & 3,40 & Seca \\
\hline
\end{tabular}

rápida evacuación de los excesos de agua que se presentaron a consecuencia de las precipitaciones. Estos resultados sugieren que contrario a lo que se pensaba, los suelos dedicados a la producción intensiva de carne en el valle medio del río Sinú, a pesar de su origen aluvial y lacustre, presentan durante la mayor parte del año condiciones que favorecen una buena aireación, y por tanto, la actividad de los organismos metanotrófos, favoreciendo así la oxidación o captura del metano.

\section{Flujo de metano}

Los resultados para el flujo de metano mostraron variaciones a lo largo del año, presentándose tanto valores negativos como positivos, lo que indica cambios en la capacidad del suelo para actuar como sumidero o emisor de metano. En general, se observó que los flujos negativos ocurrieron en los meses donde prevalecieron precipitaciones inferiores a $100 \mathrm{~mm}$ (Cuadro 3), periodo considerado como época seca. Flujos positivos se presentaron en los meses de abril, julio, agosto y noviembre de 2015, cuando las condiciones de precipitación fueron prevalentemente altas. Lo anterior puede estar asociado al desarrollo de condiciones de saturación de humedad en el suelo (Figura 3).

Los análisis de varianza realizados por separado a cada uno de los muestreos mensuales indicaron que los flujos de metano no fueron afectados por el tipo de cobertura vegetal (pastura) o el nivel de fertilización nitrogenada evaluados en estos ensayos ( $>0,05)$. Lo anterior indica que las coberturas de pasturas y los niveles de fertilización nitrogenada no afectaron los flujos de metano que se presentaron en los suelos durante esta investigación. Sin embargo, hubo una interacción estadísticamente significativa $(\mathrm{p}=0,0004)$ entre gramíneas y el nivel de fertilización nitrogenada (Figura 4). 


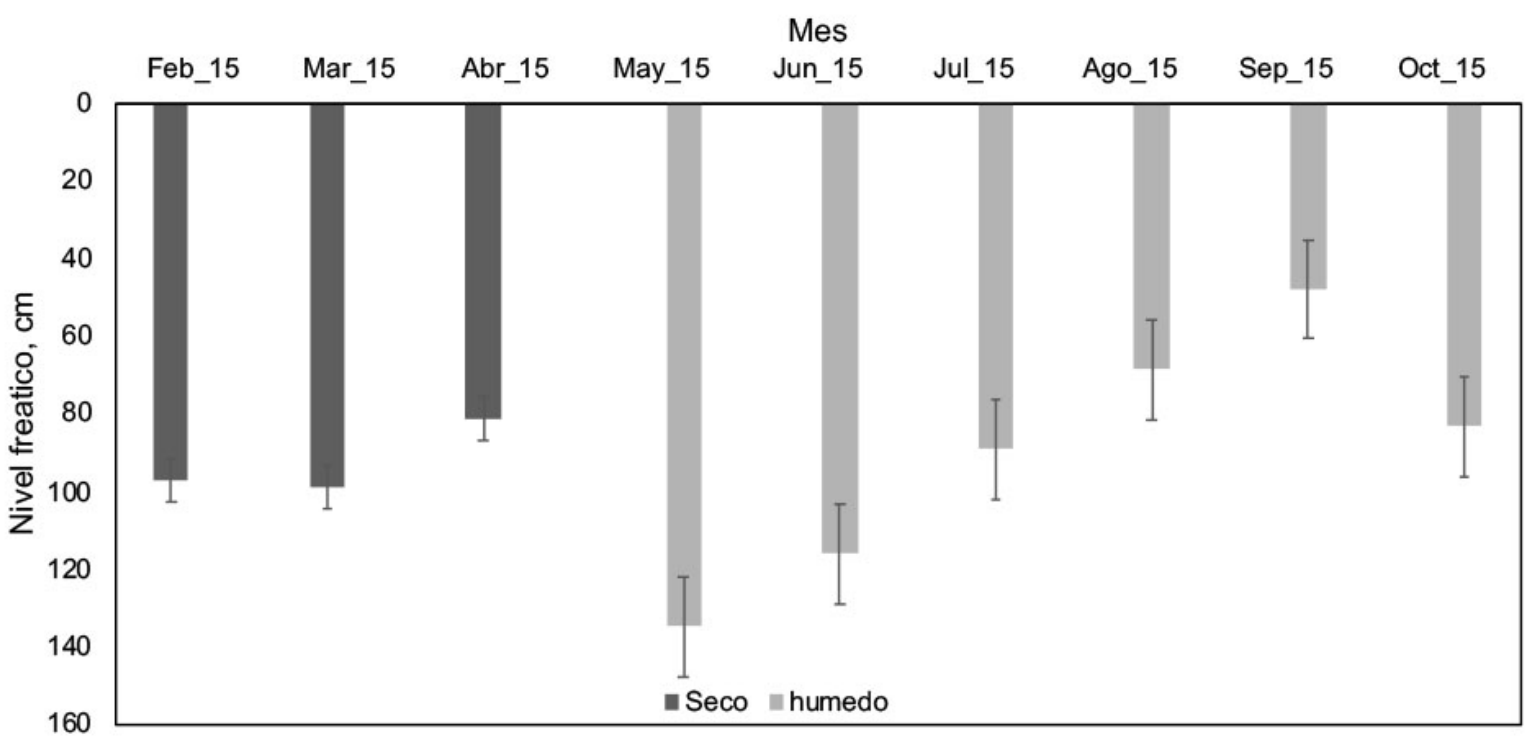

Figura 2. Comportamiento del nivel freático durante el período experimental, para monitorear los flujos de metano del suelo con diferentes coberturas de pastos (Brachiaria humidicola cv CIAT679, Panicum maximum cv Tanzania). Valle medio del río Sinú en Colombia. 2014 - 2015

Figure 2. Watertable behavior during the experimental period to monitor soil methane fluxes with different grass cover (Brachiaria humidicola cv CIAT 679, Panicum maximum cv Tanzania). Middle valley of the Sinu river in Colombia. 2014 - 2015.

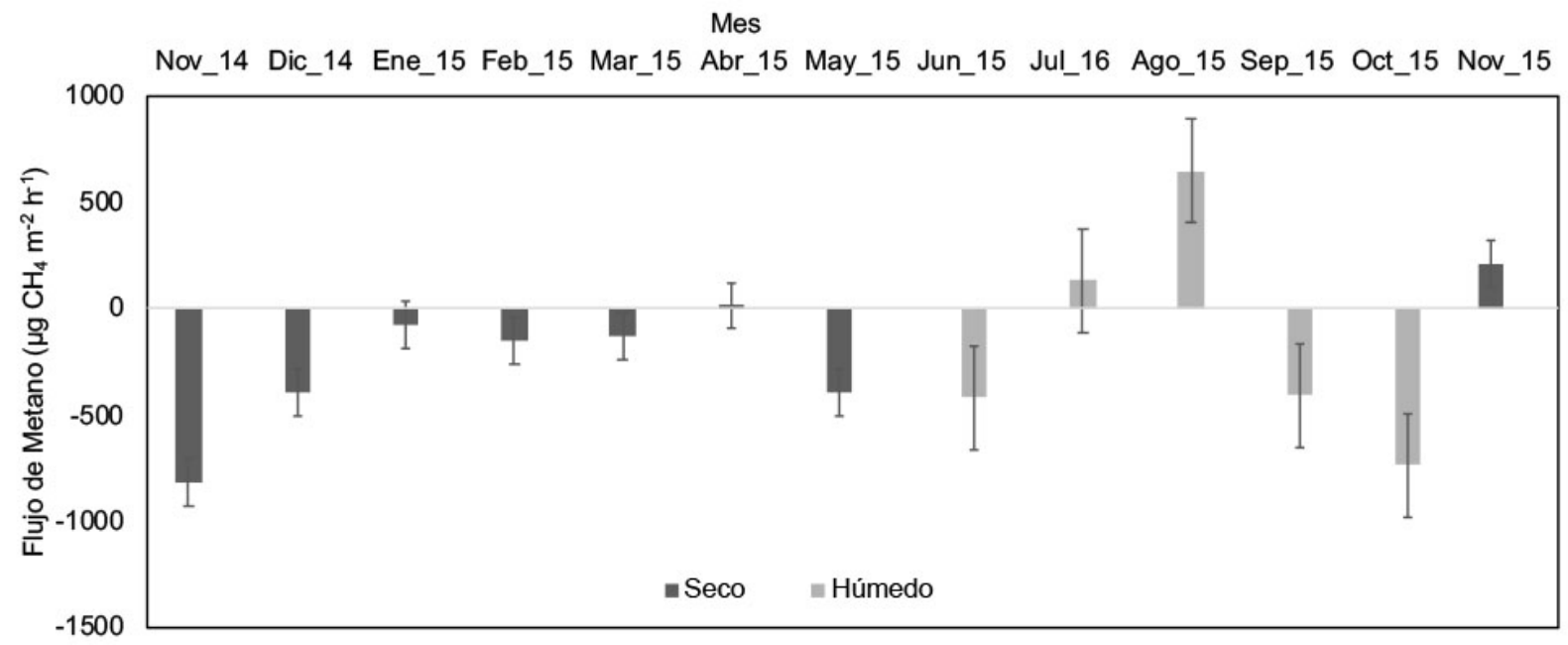

Figura 3. Flujos de metano en un período de monitoreo de 360 días en pastizales (Brachiaria humidicola cv. CIAT679, Panicum maximum cv. Tanzania), bajo un sistema de producción rotacional en el valle medio del río Sinú en Colombia. 2014 - 2015.

Figure 3. Methane fluxes in a 360-day monitoring period in pastures (Brachiaria humidicola cv. CIAT679, Panicum maximum cv. Tanzania), under a rotational production system in the middle valley of the Sinú river in Colombia. 2014 - 2015. 


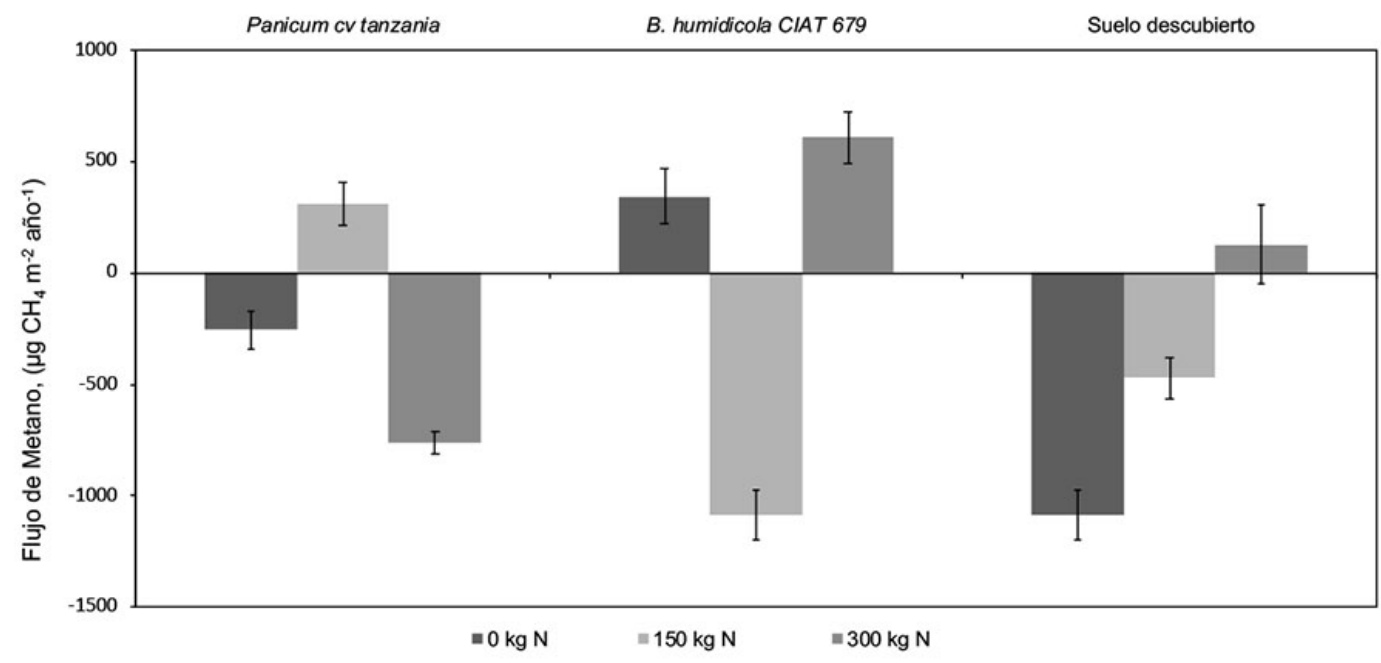

Figura 4. Flujo de metano del suelo por efecto de la interacción entre las especies de gramíneas y niveles de fertilización nitrogenada, bajo un sistema de producción rotacional en el valle medio del río Sinú en Colombia. 2014 - 2015.

Figure 4. Soil methane flow due the interaction between pasture species and nitrogen fertilization levels under a rotational production system in the Middle valley of the Sinú river in Colombia. 2014 - 2015.

\section{Variables ambientales}

En el análisis de componentes principales, las variables ambientales explicaron hasta el $60 \%$ del comportamiento de los flujos de metano. En estos análisis, se muestra que el componente 1 pudo explicar el 38,48 $\%$ de esta variación, mientras que el componente 2 explicó el 21,64 \% (Figura 5). Adicionalmente, se evidencia que las variables ambientales que explican un mejor comportamiento de los flujos de metano (primer eje) fueron la humedad relativa $(0,88)$, evaporación $(-0,83)$ y precipitación $(0,72)$.

La precipitación y humedad relativa se relacionaron de forma directa con los flujos de metano, e indicó altos flujos de metano hacia la atmósfera, debido a mayor precipitación y humedad relativa durante el período experimental. Por el contrario, la evaporación y temperatura ambiente presentaron relación inversa con el flujo de metano, lo que indica menores emisiones de este gas hacia la atmósfera a temperaturas y niveles de evaporación más altos. Este comportamiento probablemente se deba a que estas condiciones ambientales asociadas con el tipo de suelo, favorecieron el aumento en la actividad de las bacterias aeróbicas en el suelo, que incrementaron los procesos de oxidación o captura de metano.

\section{Variables fisicoquímicas del suelo}

El análisis de componentes principales componente principal sobre las propiedades físicas del suelo explicó el $70 \%$ de los flujos de metano. En este caso, el componente 1 explicó el $45 \%$ de esta variación y el componente 2 representó el $25 \%$ de esta variación (Figura 6). Dentro del primer componente, las variables que explicaron mejor el flujo de metano fueron las relacionadas con la densidad aparente $(0,94)$, la porosidad $(-0,93)$ y la humedad del suelo $(-0,66)$. En el caso del segundo componente, las variables que mejor explicaron el flujo de metano fueron la conductividad hidráulica $(-0,65)$ y el porcentaje de la porosidad ocupada por el agua $(-0,67)$. Los resultados anteriores destacan la importancia de las condiciones aeróbicas y la disponibilidad de oxígeno en el suelo que 


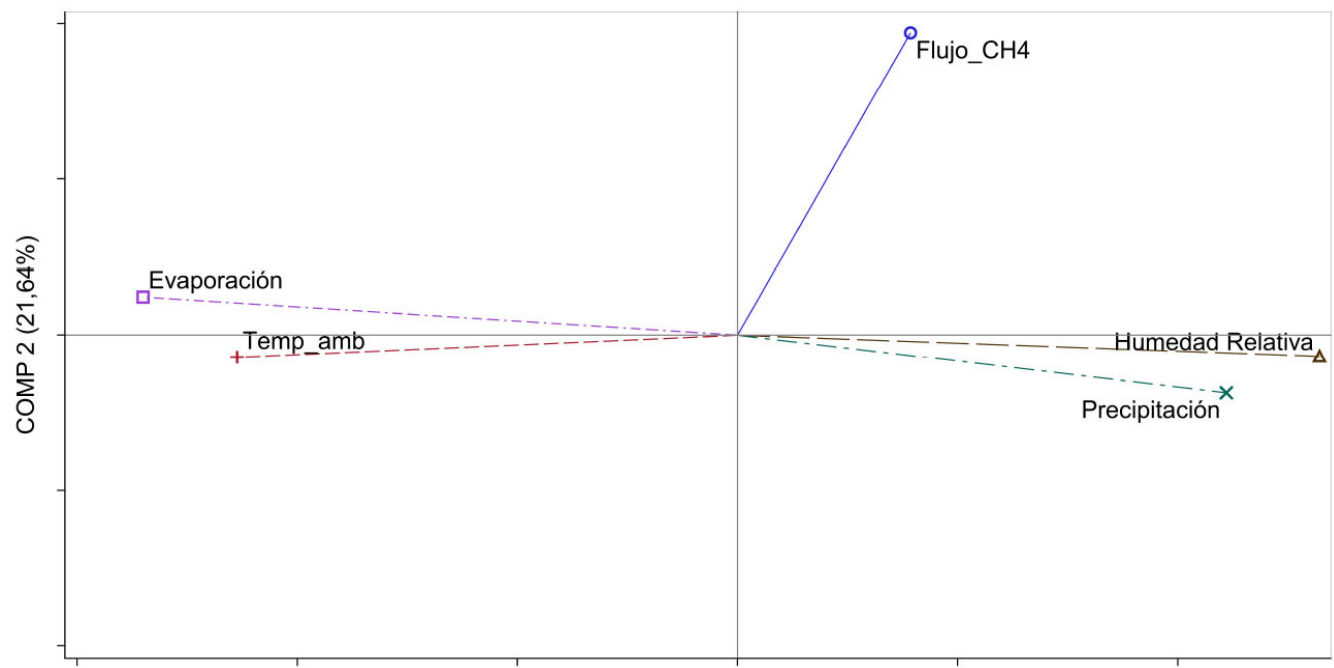

COMP $1(38,48 \%)$

Figura 5. Análisis de componentes principales entre las variables ambientales y el flujo de metano en el suelo con dos gramíneas (Brachiaria humidicola cv. CIAT679 y Panicum maximum cv. Tanzania), establecidas en el Valle medio del río Sinú, in Colombia. 2014 - 2015.

Figure 5. Principal component analysis between the environmental variables and soil methane flux in two grasses (Brachiaria humidicola cv. CIAT679 and Panicum maximum cv. Tanzania), established in the Middle valley of the Sinú river., in Colombia. 2014 $-2015$.

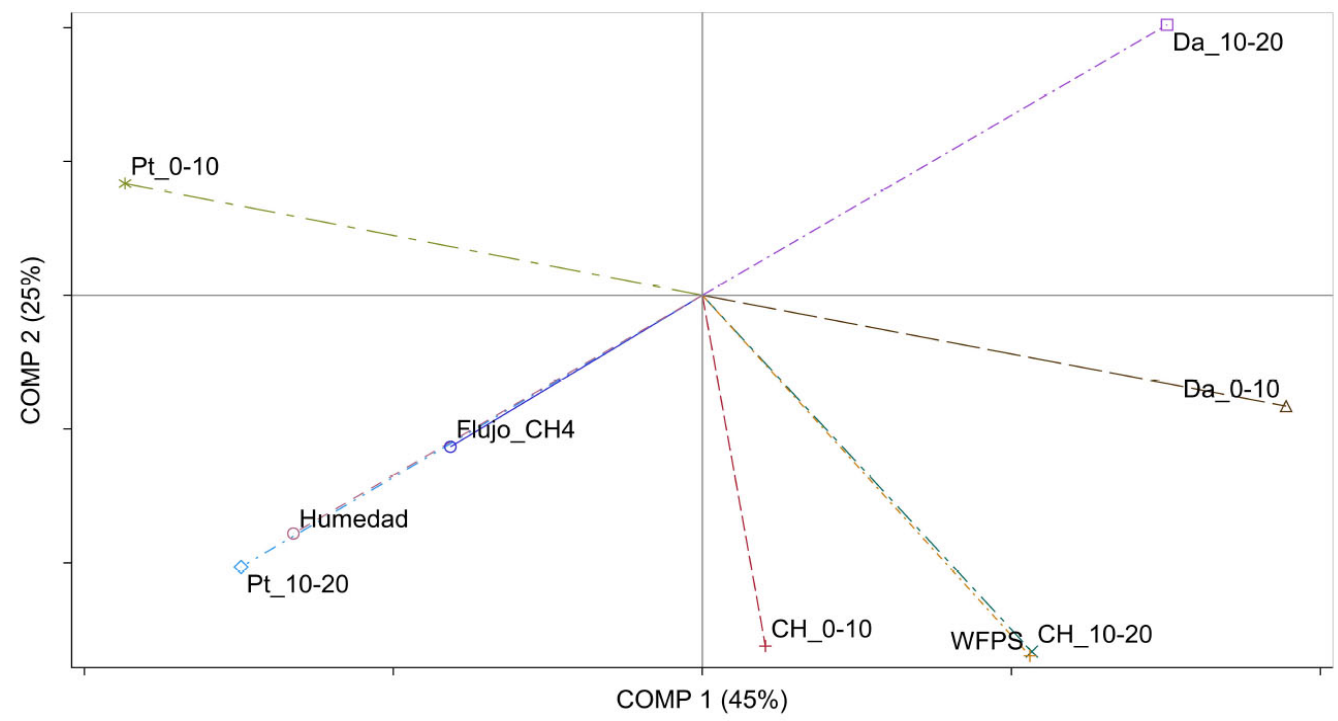

Figura 6. Análisis de componentes principales entre las propiedades físicas de suelo el flujo de metano en el suelo con dos gramíneas (Brachiaria humidicola cv. CIAT679 y Panicum maximum cv. Tanzania), establecidas en el Valle medio del río Sinú, in Colombia. $2014-2015$.

Pt: porosidad total (\%), flujo_CH4: flujo de metano $\left(\mu \mathrm{g} \mathrm{CH}_{4} \mathrm{~m}^{-2} \mathrm{hr}^{-1}\right), \mathrm{CH}$ : conductividad hidráulica saturada $\left(\mathrm{mm} \mathrm{hr}^{-1}\right)$, Da: densidad aparente del suelo $\left(\mathrm{g} \mathrm{cm}^{-3}\right)$, WFPS: porcentaje de la porosidad ocupada por agua (\%).

Figure 6. Principal component analysis between the soil physical properties and the flow of methane with two grasses (Brachiaria humidicola cv. CIAT679 and Panicum maximum cv. Tanzania), established in the Middle valley of the Sinú river, in Colombia. 2014 - 2015.

Pt: total porosity (\%), flujo_CH4: methane fluxes $\left(\mu \mathrm{g} \mathrm{CH}_{4} \mathrm{~m}^{-2} \mathrm{hr}^{-1}\right), \mathrm{CH}$ : saturated hydraulic conductivity (mm hr-1), Da: soil bulk density $\left(\mathrm{g} \mathrm{cm}^{-3}\right)$, WFPS: water filled porosity space $(\%)$. 
favorecen los procesos de actividad microbiana, oxidación y absorción de metano. Se encontró una relación directa y positiva entre los flujos de metano y la densidad aparente del suelo y la humedad del suelo, indicando que mayores flujos de metano hacia la atmósfera con valores altos de densidad aparente y humedad del suelo. De manera similar, se encontró una relación inversa con la porosidad, lo que indica flujos de metano más bajos hacia la atmósfera se relacionan con suelos con altos valores de porosidad total $(\mathrm{Pt})$.

En cuanto a las propiedades químicas del suelo, el análisis de componentes principales indicó que los flujos de metano estaban condicionados de la siguiente forma: en el componente 1 las variables que mejor los explicaron fueron el contenido de calcio intercambiable $(0,95)$, azufre $(0,94)$, conductividad eléctrica $(0,89)$ y los sulfatos $(0,80)$, entre otros. Lo anterior indicó una relación directa con calcio, azufre, conductividad eléctrica y sulfatos. En el componente 2, las variables que mejor explicaron el comportamiento de los flujos de metano fueron $\mathrm{pH}(-0,93)$, contenido de materia orgánica $(0,88)$, manganeso $(-0,66)$ y capacidad de intercambio catiónico $(0,58)$ (Figura 7$)$. $\mathrm{El} \mathrm{pH}$ del suelo presentó una relación inversa, lo que indica que, valores de $\mathrm{pH}$ más altos produjeron menos flujo de $\mathrm{CH}_{4}$ hacia la atmósfera. El azufre (S) mostró una correlación positiva significativa con los flujos de $\mathrm{CH}_{4}$, lo que indica que a mayor contenido de S, mayor potencial de oxidación de metano en el suelo (sumidero). En este sentido,

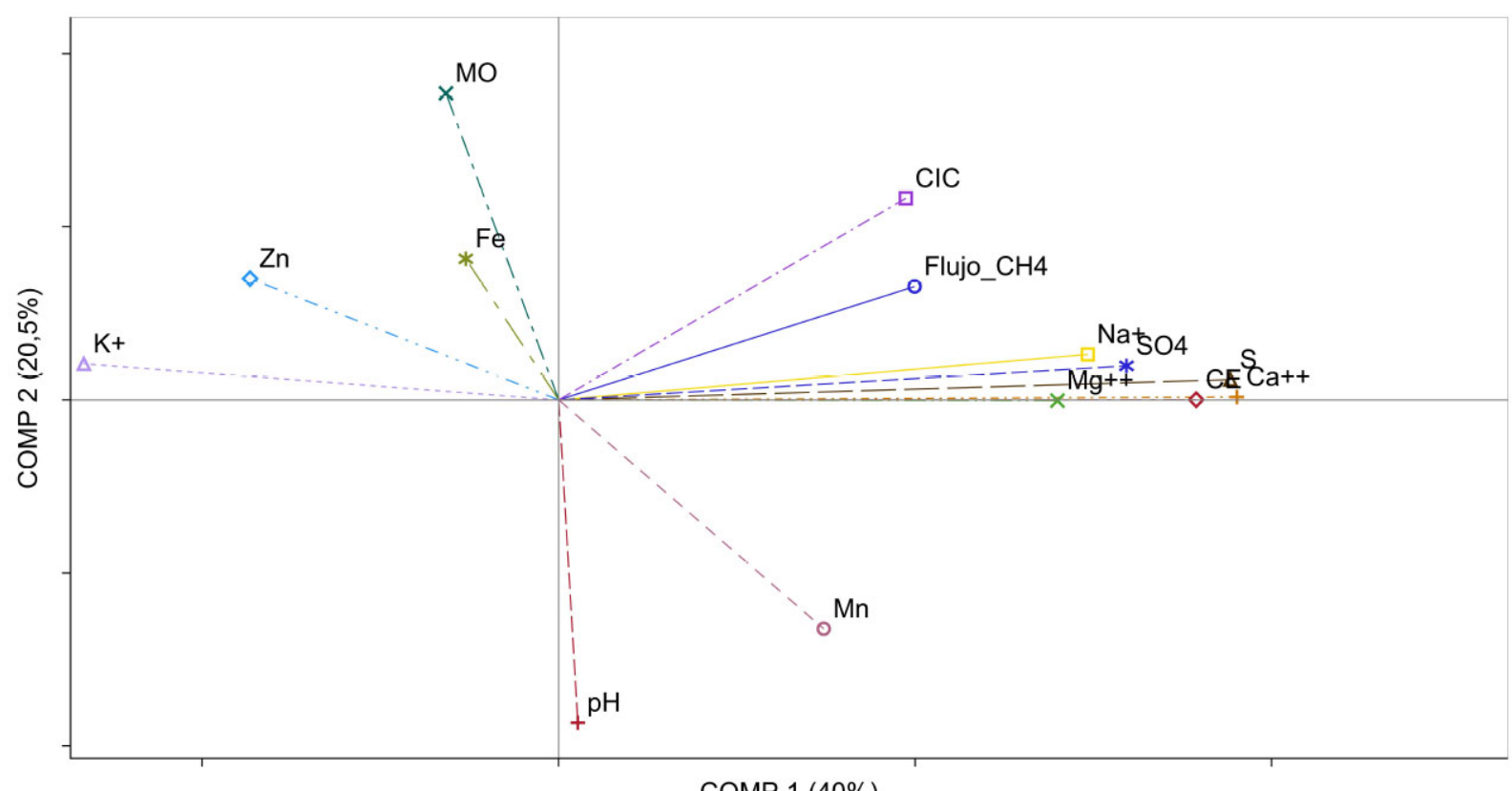

COMP $1(40 \%)$

Figura 7. Análisis de componentes principales entre las propiedades químicas de suelo el flujo de metano en el suelo con dos gramíneas establecidas (Brachiaria humidicola cv. CIAT679 y Panicum maximum cv. Tanzania), establecidas en el Valle medio del río Sinú, in Colombia. 2014 - 2015.

Flujo_CH4: flujos de metano, MO: materia orgánica (\%), pH: reacción del suelo, CE: conductividad eléctrica $\left(\mathrm{dS} \mathrm{m}^{-1}\right), \mathrm{Ca}++; \mathrm{K}+$; $\mathrm{Mg++} ; \mathrm{Na}$ : bases intercambiables $\left(\mathrm{cmol}_{(+)} \mathrm{kg}^{-1}\right)$, CIC: capacidad de intercambio catiónico $\left(\mathrm{cmol}_{(+)} \mathrm{kg}^{-1}\right), \mathrm{S}:$ azufre $\left(\mathrm{mg} \mathrm{kg}^{-1}\right), \mathrm{Fe} ; \mathrm{Mn}$; $\mathrm{Zn}$ : elementos menores del suelo $\left(\mathrm{mg} \mathrm{kg}^{-1}\right), \mathrm{SO}_{4}$ : sulfatos.

Figure 7. Principal component analysis between the soil chemical properties and soil methane flux in two grasses established (Brachiaria humidicola cv. CIAT679 and Panicum maximum cv. Tanzania), in the Middle valley of the Sinú river, in Colombia. 2014 - 2015.

Flujo_CH4: methane fluxes, MO: organic matter (\%), pH: soil reaction, CE: electrical conductivity (dS m$\left.{ }^{-1}\right), \mathrm{Ca}^{++} ; \mathrm{K}^{++} \mathrm{Mg}^{++} ; \mathrm{Na}^{+}$: exchangeable bases $\left(\mathrm{cmol}_{(+)} \mathrm{kg}^{-1}\right)$, CIC: cation exchange capacity $\left(\mathrm{cmol}_{(+)} \mathrm{kg}^{-1}\right)$, S: sulfur $\left(\mathrm{mg} \mathrm{kg}^{-1}\right), \mathrm{Fe}$; Mn; Zn: minor soil elements $\left(\mathrm{mg} \mathrm{kg}^{-1}\right), \mathrm{SO}_{4}$ : sulfates. 
algunos autores afirmaron que el contenido de sulfato $\left(\mathrm{SO}_{4}\right)$ en el suelo favorece la acumulación de $\mathrm{CH}_{4}$ (sumidero), debido al aumento de la actividad de las bacterias reductoras de $\mathrm{SO}_{4}$ (Reay et al., 2007).

\section{Discusión}

Los resultados obtenidos en este experimento mostraron que los suelos en el área de estudio se comportaron como sumideros de metano la mayor parte del año, incluso en algunos períodos de la temporada de lluvias. Esto sugiere que los suelos en estudio dedicados a la producción ganadera en el valle medio del río Sinú presentaron buenas condiciones de drenaje y propiedades físicas de suelo que favorecieron la aireación y la actividad de microorganismos metanotrófos (Merino et al., 2004). Se encontró que el nivel freático estuvo por encima de los $50 \mathrm{~cm}$ de profundidad la mayor parte del período experimental, lo que indica que la mayor parte del año el suelo en estudio no presentó condición de reducción a causa del exceso de humedad o baja disponibilidad de oxígeno. Resultados similares reportaron Martínez-Atencia et al. (2020), bajo un sistema silvopastoril continuo al área estudió, donde se realizó monitoreo del nivel freático, encontrado que gran parte del año el nivel freático estuvo sobre los $40 \mathrm{~cm}$ de profundidad.

De acuerdo con los resultados obtenidos en el análisis de componentes principales, las variables físicas de suelo: densidad aparente, porosidad y humedad del suelo fueron las responsables de explicar la mayor parte de las variaciones en los flujos de metano. Estos resultados sugieren que los flujos de metano negativos se ven favorecidos por las condiciones aeróbicas y disponibilidad de oxígeno en los suelos, lo que favoreció la actividad de los microorganismos metanotrófos.

No se encontraron influencias de las coberturas con pasturas y los niveles de fertilización nitrogenada con respecto a los flujos de metano, con pocos efectos de estas variables sobre los flujos de este gas en el suelo.

Con estos hallazgos, contrario a lo que se pensaba, los suelos en el valle medio del río Sinú en Colombia, sobre los que se realizó la presente evaluación, a pesar de su origen aluvial y fluvio-lacustre, presentaron condiciones adecuadas de drenaje y porosidad que favorecieron condiciones para obtener un balance negativo en el flujo o captura de metano, comportamiento que se registró durante la mayor parte del año. Las precipitaciones inferiores a $100 \mathrm{~mm}$ por mes, que se presentaron durante los años 2014 y 2015, favorecieron la adecuada aireación del suelo la mayor parte del año. De igual forma, debido a que la temperatura del suelo se mantuvo en el rango de 25 a $35^{\circ} \mathrm{C}$ durante todo el año, se pudo haber favorecido la actividad microbiana aeróbica. Sin embargo, en el sitio donde se ubican estos suelos, ocurren periodos del año durante los cuales la saturación del agua del suelo también puede ocasionar la emisión de metano hacia la atmósfera, en especial en los meses donde la precipitación es alta (julio y agosto).

Los flujos de metano, bajo la interacción de las coberturas y niveles de fertilización nitrogenada que se muestran en la Figura 4, evidencian variabilidad en la información. Los resultados obtenidos no definieron tendencia en su comportamiento, lo que no permitió inferir sobre la interacción de las gramíneas y niveles de fertilización encontrada.

Se observó dentro de estos resultados que en el suelo descubierto, asociado a los niveles de fertilización, los flujos de metano dependieron de las cantidades de fertilización, nitrogenada por año, en dosis inferiores a $150 \mathrm{~kg}$ $\mathrm{N} \mathrm{ha}^{-1}$ año $\mathrm{O}^{-1}$, el suelo se comportó como sumidero de metano $\left(-810 \mu \mathrm{g}\right.$ de $\left.\mathrm{CH}_{4} \mathrm{~m}^{-2} \mathrm{~h}^{-1}\right)$; dosis superiores a $150 \mathrm{~kg}$ $\mathrm{N} \mathrm{ha}^{-1}$ año ${ }^{-1}$, se inhibió la actividad de los microrganismos metanotrófos en el suelo, generando emisiones de $\mathrm{CH}_{4}$ hacia la atmósfera.

Los resultados del análisis de correlación entre las propiedades físicas del suelo y los flujos de metano (Figura 6) mostraron una relación directa y positiva, lo que indicó flujos de metano más altos hacia la atmósfera con mayor densidad aparente y humedad del suelo. De manera similar, se encontró una relación inversa con la porosidad, lo que indicó flujos de metano más bajos hacia la atmósfera a valores más altos de porosidad. Diferentes estudios al respecto sugirieron que el aumento en la densidad aparente es un indicativo de la compactación y el deterioro 
del suelo, disminuye los espacios porosos y reduce el proceso de oxidación del metano en el suelo (Hansen et al., 1993; Ruser et al., 1998; Sitaula et al., 2001; Fujikawa y Miyazaki, 2005; Gebert et al., 2011). Esto significa que suelos bien aireados, con alta porosidad y texturas francas tienen potencial de capturar metano, debido a las buenas condiciones físicas los excesos de agua son drenados, lo que favorece la disponibilidad de oxígeno en la actividad de los microorganismos metanótrofos y los procesos de oxidación del metano (Born et al., 1990; Dörr et al., 1993; Ferreira, 2008).

En la mayoría de los ecosistemas naturales y agrícolas, la variación en la humedad del suelo afecta los procesos oxidativos (actividad bacterias aeróbicas), los cuales se ven favorecidos por la escasez de lluvias o períodos prolongados de verano, conllevando a la absorción o captura de metano (Thangarajan et al., 2013; Palm et al., 2014). En los suelos del valle medio del río Sinú, dedicados a la producción ganadera, las condiciones favorecieron la metanogénesis entre julio y octubre, pero las precipitaciones inferiores a $100 \mathrm{~mm}$, favorecieron el desarrollo de condiciones aeróbicas y acción de microorganismos metanotrófos, lo que puede explicar el comportamiento de los flujos de metano en estos suelos.

$\mathrm{El} \mathrm{pH}$ del suelo presentó una relación inversa (Figura 7), lo que indicó que suelos con pH altos (bajas concentraciones de iones $\mathrm{H}^{+}$), produjeron menos metano hacia la atmósfera; esto sugiere que el proceso de oxidación de metano se vio favorecido por valores de $\mathrm{pH}$ cercanos a 7, reduciéndose las emisiones hacia la atmósfera. En este sentido, algunos autores afirmaron, que la acidificación excesiva del suelo (altas concentraciones de iones $\mathrm{H}^{+}$) o los altos niveles de aluminio $(\mathrm{Al})$ son capaces de inhibir la actividad de las bacterias metanotróficas responsables de la oxidación del metano (Nanba y King, 2000; Bradford et al., 2001; Sitaula et al., 2001; Dunfield, 2007).

\section{Conclusiones}

Los resultados de la presente investigación permitieron concluir que los flujos de metano en suelos dedicados a la producción intensiva de carne en el valle medio del río Sinú no fueron afectados por el tipo de cobertura (pastura), ni por la fertilización nitrogenada aplicada para incrementar las producciones de alimento. Se creía que por su origen y características de drenaje, estos suelos podrían estar favoreciendo la actividad de microorganismos metanogénicos, así como la liberación de metano hacia la atmosfera. Sin embargo, se encontró que los flujos de metano variaron a lo largo del año y dependieron de las condiciones de humedad del suelo, en relación con el comportamiento del nivel freático y la precipitación. Las propiedades físicas de los suelos en el valle medio del río Sinú favorecieron condiciones de aireación y captura de metano durante la mayor parte del año, pero se presentaron aumento de la liberación de $\mathrm{CH}_{4}$ hacia la atmósfera en periodos de lluvias intensas. Las condiciones ambientales del valle medio del río Sinú asociadas con las características físicas del suelo (texturas, aireación y condiciones adecuadas de drenaje), favorecieron los procesos de oxidación de metano durante la mayor parte del año. La metanogénesis puede ocurrir en períodos cuando las precipitaciones son excesivas y las condiciones de aireación del suelo se reducen, lo cual ocurrió especialmente en los meses de julio y agosto.

\section{Agradecimientos}

Los autores expresan su gratitud con la Corporación Colombiana de Investigación Agropecuaria (AGROSAVIA) y el Ministerio de Agricultura y Desarrollo Rural (MADR) por financiar el proyecto "Producción intensiva de carne en pasturas con diferente capacidad para inhibir la nitrificación y reducir las emisiones de gases de efecto invernadero" bajo de la cual se obtuvo este documento, con el acuerdo de colaboración No. 054/2008. 


\section{Literatura citada}

Aronson, E.L., and B.R. Helliker. 2010. Methane flux in non-wetland soils in response to nitrogen addition: a meta-analysis. Ecology 91:3242-3251. doi:10.1890/09-2185.1

Bernabé, Y., and A. Maineult. 2015. Physics of porous media: fluid flow through porous media. In: G. Schubert, editor, Treatise on geophysics. $2^{\text {nd }}$ ed. Vol 11. Resources in near-surface earth. Elsevier, Amsterdam, NLD. p. 19-41. doi:10.1016/b978$0-444-53802-4.00188-3$

Boeckx, P., O. Cleemput, and I. Cillaralvo. 1997. Methane oxidation in soils with different textures and land use. Nutrient Cycling Agroecosyst. 49:91-95. doi:10.1023/A:1009706324386

Born, M., H. Dörr, and I. Levin. 1990. Methane consumption in aerated soils of the temperate zone. Tellus 42(1):2-8. doi:10.1034/j.1600-0889.1990.00002.x

Bradford, M., P. Ineson, P. Wookey, and H. Lappin-Scott. 2001. The effects of acid nitrogen and acid sulphur deposition on $\mathrm{CH}_{4}$ oxidation in a forest soil: a laboratory study. Soil Biol. Biochem. 33:1695-1702. doi:10.1016/s0038-0717(01)00091-8

Burt, R. 2014. Soil survey field and laboratory methods manual. Soil Survey Investigations Report No. 51, Version 2. US Department of Agriculture, Natural Resources Conservation Service, WA, USA.

Capa, M.E.D. 2015. Efecto de la fertilización orgánica y mineral en las propiedades del suelo, la emisión de los principales gases de efecto invernadero y en las diferentes fases fenológicas del cultivo de café (Coffea arabica L.). Tesis Ph.D., Universidad de Madrid, Madrid, ESP.

Castro, M.S., H.L. Gholz, K.L. Clark, and P.A. Steudler. 2000. Effects of forest harvesting on soil methane fluxes in Florida slash pine plantations. Can. J. For. Res. 30:1534-1542. doi:10.1139/cjfr-30-10-1534

Charmley, E., S.R.O. Williams, P.J. Moate, R.S. Hegarty, R.M. Herd, V.H. Oddy, P. Reyenga; K.M. Staunton, A. Anderson, and M.C. Hannah. 2016. A universal equation to predict methane production of forage-fed cattle in Australia. Anim. Prod. Sci. 56(3):169-180. doi:10.1071/an15365

Chu, H., Y. Hosen, and K. Yagi. 2007. $\mathrm{NO}, \mathrm{N}_{2} \mathrm{O}, \mathrm{CH}_{4}$ and $\mathrm{CO}_{2}$ fluxes in winter barley field of Japanese Andisol as affected by N fertilizer management. Soil Biol. Biochem. 39:330-339. doi:10.1016/j.soilbio.2006.08.003

Clark, H. 2016. The estimation and mitigation of agricultural greenhouse gas emissions from livestock. In: D. Yulistiani, editors, Proceedings of International Seminar on Livestock Production and Veterinary Technology. Indonesian Agency for Agricultural Research Development, IDN. p. 5-13. doi:10.14334/proc.intsem.lpvt-2016-p.5-13

Di-Rienzo, J., F. Casanoves, M. Balzarina, L. Gonzalez, M. Tablada, y C. Robledo. 2018. Infostat versión 2018. Universidad Nacional de Córdoba, ARG.

Dörr, H., L. Katruff, and I. Levin. 1993. Soil texture parameterization of the methane uptake in aerated soils. Chemosphere 26:697-713. doi:10.1016/0045-6535(93)90454-d

Dunfield, P.F. 2007. The soil methane sink. In: D.S. Reay et al., editors, Greenhouse gas sinks. CABI, Wallingford, USA, and Cambridge, GBR.p. 152-170.

Fang, H., S. Cheng, G. Yu, J. Cooch, Y. Wang, M. Xu, and Y. Li. 2014. Low-level nitrogen deposition significantly inhibits methane uptake from an alpine meadow soil on the Qinghai-Tibetan Plateau. Geoderma 213:444-452. doi:10.1016/j. geoderma.2013.08.006

FAO. 2018. Soluciones ganaderas para el cambio climático. FAO, Roma, ITA. 
Ferreira, O. 2008. Flujos de gases de efecto invernadero, potencial de calentamiento global y evaluación de emergía del sistema agroforestal Quesungual en el sur de Lempira, Honduras. Tesis MSc., Universidad Nacional de Colombia, Palmira, COL.

Fujikawa, T., and T. Miyazaki. 2005. Effects of bulk density and soil type on the gas diffusion coefficient in repacked and undisturbed soils. Soil Sci. 170:892-901. doi:10.1097/01.ss.0000196771.53574.79

Gebert, J., A. Groengroeft, and E. Pfeiffer. 2011. Relevance of soil physical properties for the microbial oxidation of methane in landfill covers. Soil Biol. Biochem. 43:1759-1767. doi:10.1016/j.soilbio.2010.07.004

Guzmán, D., J.F. Ruiz, y M. Cadena. 2014. Regionalización de Colombia según la estacionalidad de la precipitación media mensual, a través análisis de componentes principales (ACP). Informe Técnico. IDEAM, Bogotá D.C., COL.

Hansen, S., J.E. Mæhlum, and L.R. Bakken. 1993. $\mathrm{N}_{2} \mathrm{O}$ and $\mathrm{CH}_{4}$ fluxes in soil influenced by fertilization and tractor traffic. Soil Biol. Biochem. 25:621-630. doi:10.1016/0038-0717(93)90202-m

Hao, W.M., D. Scharffe, P.J. Crutzen, and E. Sanhueza. 1988. Production of $\mathrm{N}_{2} \mathrm{O}, \mathrm{CH}_{4}$, and $\mathrm{CO}_{2}$ from soils in the tropical savanna during the dry season. J. Atmos. Chem. 7:93-105. doi:10.1007/bf00048256

Hernández-Medrano, J.H., y L. Corona. 2018. El metano y la ganadería bovina en México: ¿Parte de la solución y no del problema? Agroproductividad 11(2):46-51.

Holdridge, L.R. 2000. Ecología basada en zonas de vida. Quinta reimpresión. IICA, San José, CRI.

Hou, A.X., G.X. Chen, Z.P. Wang, O. Van-Cleemput, and W.H. Patrick. 2000. Methane and nitrous oxide emissions from a rice field in relation to soil redox and microbiological processes. Soil Sci. Soc. Am. J. 64:2180-2186. doi:10.2136/ sssaj2000.6462180x

Hristov, A.N., J. Oh, C. Lee, R. Meinen, F. Montes, T. Ott, J. Firkins, A. Rotz, C. Dell, A. Adesogan, W. Yang, J. Tricarico, E. Kebreab, G. Waghorn, J. Dijkstra and S. Oosting. 2013. Mitigation of greenhouse gas emissions in livestock production - A review of technical options for non- $\mathrm{CO}_{2}$ emissions. FAO Animal Production and Health Paper No. 177. FAO, Rome, ITA.

IGAC (Instituto Geográfico Agustín Codazzi). 2006. Métodos analíticos del laboratorio de suelos. $6^{\mathrm{a}}$ ed. Imprenta Nacional de Colombia, Bogotá, COL.

Kammann, C., L. Grünhage, H.J. Jäger, and G. Wachinger. 2001. Methane fluxes from differentially managed grassland study plots: the important role of $\mathrm{CH}_{4}$ oxidation in grassland with a high potential for $\mathrm{CH}_{4}$ production. Environ. Poll. 115:261273. doi:10.1016/s0269-7491(01)00103-8

Kähkönen, M.A., C. Wittmann, H. Ilvesniemi, C.J. Westman, and M. Salkinoja-Salonen. 2002. Mineralization of detritus and oxidation of methane in acid boreal coniferous forest soils: seasonal and vertical distribution and effects of clear-cut. Soil Biol. Biochem. 34:1191-1200. doi:10.1016/s0038-0717(02)00056-1

Klemedtsson, A.K., and L. Klemedtsson. 1997. Methane uptake in Swedish forest soil in relation to liming and extra N-deposition. Biol. Fert. Soils 25:296-301. doi:10.1007/s003740050318

Lassey, K.R. 2008. Livestock methane emission and its perspective in the global methane cycle. Aust. J. Exp. Agr. 48:114-118. doi:10.1071/ea07220

Lê, S., J.J, and F. Husson. 2008. FactoMineR: A Package for Multivariate Analysis. J. Stat. Softw. 25(1):1-18. doi:10.18637/ jss.v025.i01

Lee, S.W., J. Im, A.A. DiSpirito, L. Bodrossy, M.J. Barcelona, and J.D. Semrau. 2009. Effect of nutrient and selective inhibitor amendments on methane oxidation, nitrous oxide production, and key gene presence and expression in landfill cover 
soils: characterization of the role of methanotrophs, nitrifiers, and denitrifiers. Appl. Microbiol. Biotechnol. 85:389-403. doi:10.1007/s00253-009-2238-7

Li, X., H. He, W. Yuan, L. Li, W. Xu, W. Liu, and Z. Wang. 2018. Response of soil methane uptake to simulated nitrogen deposition and grazing management across three types of steppe in Inner Mongolia, China. Sci. Total Environ. 612:799808. doi:10.1016/j.scitotenv.2017.08.236

Martínez-Atencia, J., J.C. Loaiza-Usuga, N.W. Osorio-Vega, G. Correa-Londoño, and M. Casamitjana-Causa. 2020. Leaf litter decomposition in diverse silvopastoral systems in a neotropical environment. J. Sustain. For. 2020:1723112. doi:10.108 $0 / 10549811.2020 .1723112$

Merino, A., P. Pérez-Batallón, y F. Macías. 2004. Influencia del uso y manejo agrícola sobre la dinámica de $\mathrm{CH}_{4}$ del suelo en el norte de España. Edafología 11(2):207-219.

Montenegro, J., y S. Abarca. 2000. Fijación de carbono, emisión de metano y de óxido nitroso en sistemas de producción bovina en Costa Rica. FAO, Roma, ITA. http://www.fao.org/3/x6366s10.htm (consultado 01 nov. 2018).

Mora, M.A., L. Ríos, L. Ríos, y J.L. Almario Charry. 2017. Impacto de la actividad ganadera sobre el suelo en Colombia. Ing. Región 17:1-12. doi:10.25054/22161325.1212

Murgueitio, E. 2011. Retos y progresos de la ganadería sostenible. Agric. Sosten. 7:45-54.

Murgueitio, E., e I. Muhammad. 2009. Ganadería y medio ambiente en América Latina En: E. Murgueitio et al., editores, Ganadería del futuro: Investigación para el desarrollo. 2ª ed. Fundación CIPAV, Cali, COL. p. 19-40.

Nanba, K., and G.M. King. 2000. Response of atmospheric methane consumption by maine forest soils to exogenous aluminum salts. Appl. Environ. Microbiol. 66:3674-3679. doi:10.1128/aem.66.9.3674-3679.2000

Pachauri, R.K., L.A. Meyer, y T. Stocker. (Ed.) 2014. IPCC 2014: Cambio climático 2014: Informe de síntesis. Contribución de los Grupos de Trabajo I. II y III al Quinto Informe de evaluación del panel intergubernamental sobre el cambio climático. IPCC, Ginebra, CHE.

Palm, C., H. Blanco-Canqui, F. DeClerck, L. Gatere, and P. Grace. 2014. Conservation agriculture and ecosystem services: An overview. Agric. Ecosyst. Environ. 187:87-105. doi:10.1016/j.agee.2013.10.010

Pereyra, V. 2009. Emisiones de metano y óxido nitroso en arrozales de la zona este del Uruguay: el manejo de cultivo como factor determinante. Repositorio Colibrí, Universidad de la República de Uruguay, Montevideo, URY. https://www. colibri.udelar.edu.uy/jspui/bitstream/20.500.12008/1456/1/uy24-14330.pdf (consultado 21 jun. 2019).

Pinheiro, J., D. Bates, S. DebRoy, D. Sarkar, and R Core Team. 2018. Nlme: linear and nonlinear mixed effects models. R package version 3.1-137. https://CRAN.R-project.org/package=nlme (accessed Jun. 21, 2019).

R Core Team. 2018. R: A language and environment for statistical computing. R Foundation for Statistical Computing, Vienna, Austria. Versión 3.4.4.

Rondón, M. 2000. Land use and balances of greenhouse gases in Colombian Tropical savannas. Ph.D. Thesis. Cornell University, NY, USA.

Reay, D.S., A.K. Smith, and N.C. Hewitt. 2007. Methane: importance, sources and sinks. In: D.S. Reay et al., editors, Greenhouse gas sinks. CABI, Wallingford, WA, USA, and Cambridge, GBR. p. 143-151. doi:10.1079/9781845931896.0143

Ruser, R., R. Schilling, H. Steindl, H. Flessa, and F. Beese. 1998. Soil compaction and fertilization effects on nitrous oxide and methane fluxes in potato fields. Soil Sci. Soc. Am. J. 62:1587-1595. doi:10.2136/sssaj1998.03615995006200060016x

Sanhueza, E. 2007. Methane, soil-vegetation-atmosphere fluxes in tropical ecosystems. Interciencia 32(1):31-34. 
Sanhueza, E., L. Cárdenas, L. Donoso, and M. Santana. 1994. Effect of plowing on $\mathrm{CO}_{2}, \mathrm{CO}, \mathrm{CH}_{4}, \mathrm{~N}_{2} \mathrm{O}$, and $\mathrm{NO}$ fluxes from tropical savannah soils. J. Geophys. Res. 99:16429-16434. doi:10.1029/94jd00265

Sanhueza, E., and L. Donoso. 2006. Methane emission from tropical savannah Trachypogon sp. grasses. Atmos. Chem. Physics 6:5315-5319. doi:10.5194/acp-6-5315-2006

Scharffe, D., M. Hao, L. Donoso, J. Crutzen, and E. Sanhueza. 1990. Soil fluxes and atmospheric concentration of CO and $\mathrm{CH}_{4}$ in the northern part of the Guayana Shield, Venezuela. J. Geophys. Res. 95:22475-22480. doi:10.1029/jd095id13p22475

Sitaula, B.K., J.I.B. Sitaula, A. Aakra, and L.R. Bakken. 2001. Nitrification and methane oxidation in forest soil: acid deposition, nitrogen input and plant effects. Water Air Soil Poll. 130:1061-1066. doi:10.1007/978-94-007-0810-5_24

USDA. 2014. Claves para la taxonomía de suelos. 12 ed. USDA, WA, USA.

Thangarajan, R., N.S. Bolan, G. Tian, R. Naidu, and A. Kunhikrishnan. 2013. Role of organic amendment application on greenhouse gas emission from soil. Sci. Total Environ. 465:72-96. doi:10.1016/j.scitotenv.2013.01.031

US EPA (US Environmental Protection Agency). 2017. Inventory of US greenhouse gas emissions and sinks 1990-2015. EPA, USA. https://www.epa.gov/sites/production/files/2017-02/documents/2017_complete_report.pdf (accessed May 29, 2019).

Visscher, A., P. Boeckx, and O. Van-Cleemput. 2007. Artificial methane sinks. In: D.S. Reay et al., editors, Greenhouse gas sinks. CABI, Wallingford, WA, USA, and Cambridge, GBR. p. 184-200. doi:10.1079/9781845931896.0184

Yang, X., C. Wang, and K. Xu. 2017. Response of soil $\mathrm{CH}_{4}$ fluxes to stimulated nitrogen deposition in a temperate deciduous forest in northern China: A 5-year nitrogen addition experiment. Eur. J. Soil Biol. 82:43-49. doi:10.1016/j.ejsobi.2017.08.004 